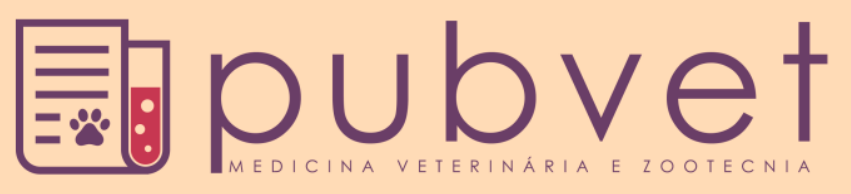

HTTP://DX.DOI.ORG/10.22256/PUBVET.V11N1.35-39

\title{
Alterações laboratoriais renais em cães com leishmaniose visceral naturalmente infectados
}

\author{
Cauê Soares Câmara1*, Pollyana Irene Baltazar², Bruno Spíndola Garcez ${ }^{3}$ \\ ${ }^{1}$ Médico veterinário e mestre em ciência animal pela Universidade Federal do Piauí, centro de ciências agrárias, Teresina- \\ PI Brasil.E-mail: caue.camara@hotmail.com \\ ${ }^{2}$ Médica veterinária e mestre em ciência animal pela Universidade Federal do Piauí, centro de ciências agrárias, Teresina- \\ PI Brasil.E-mail:polinhavet@hotmail.com \\ ${ }^{3}$ Programa de Pós-Graduação em Ciência Animal, Universidade Federal do Piauí, centro de ciências agrárias, Teresina-PI \\ Brasil.E-mail: brunosgarcez@veterinario.med.br \\ *Autor para correspondência
}

RESUMO. A Leishmaniose Visceral Canina (LVC) é uma zoonose de grande relevância no Brasil acarretando na maioria das vezes uma doença renal que pode ser a única manifestação em cães com LVC, podendo progredir até o estágio de falência renal. Foram utilizados 12 cães, sem raça definida (SRD), machos divididos em 2 grupos. Com o primeiro formado por cães com sorologia e parasitologia negativas para LVC e o segundo, positivos. Foram coletadas amostras de sangue dos animais selecionados para análise bioquímica de ureia, creatinina, proteína total e globulina, além de amostras de urina para realização da urinálise. Hiperproteinemia com hiperglobulinemia, bem como a presença marcante de albumina na urina foram achados relevantes nos cães positivos. Foi verificado significativo nos valores de ureia e creatinina, como também, na concentração de proteína da urina dos animais positivos. As alterações bioquímicas observadas mostraram concordância com os resultados encontrados na literatura, demonstrando que em pacientes positivos para LVC a disfunção renal é de grande importância para auxiliar no estadiamento clínico.

Palavras chave: azotemia, disfunção renal, leishmaniose

\section{Renal laboratory abnormalities in dogs with visceral leishmaniasis naturally infected}

\begin{abstract}
The Canine Visceral Leishmaniasis (CVL) is a zoonosis of great relevance in Brazil resulting in most of the times a kidney disease that can be the only manifestation in dogs with CVL and can progress to the stage of renal failure. 12 dogs were used, mongrel dogs, males divided into 2 groups. The first formed by negative dogs with serology and parasitology for CVL and the second positive. Animal blood samples were collected for biochemical analysis selected from urea, creatinine, total protein and globulin, and urine samples for urinalysis of. Hyperproteinaemia to hyperglobulinemia, as well as increased presence of albumin in urine were found in the relevant positive dogs. It was observed in the mean values of urea and creatinine, as well as, the protein concentration in urine of positive animals. The biochemical changes observed showed agreement with the results found in the literature, demonstrating that in patients positive for CVL renal dysfunction is of great importance to aid in the clinical staging.
\end{abstract}

Keywords: azotemia, renal dysfunction, leishmaniosis 


\title{
Alteraciones de laboratorio renales en perros naturalmente infectados con leishmaniosis visceral
}

\begin{abstract}
RESUMEN. La Leishmaniosis Visceral Canina (LVC) es una zoonosis de grande relevancia en Brasil acarreando en la mayoría de las veces una enfermedad renal que puede ser la única manifestación en perros con LVC, pudiendo evolucionar hasta la falencia renal. Fueron utilizados 12 perros criollos, machos divididos en 2 grupos. El primero formado por perros con serología y parasitología negativas para LVC y el segundo, positivos. Fueron colectadas muestras de sangre de los animales seleccionados para análisis bioquímica de urea, creatinina, proteína total y globulina, además de muestras para realización análisis de orina. Hiperproteinemia con hiperglobulinemia, bien como la destacada presencia de albumina en la orina fueron encontrados relevantes en los perros positivos. Fue verificado significativo en los valores de urea y creatinina, como también, en la concentración de proteína en la orina de los animales positivos. Las alteraciones bioquímicas observadas mostraron concordancia con los resultados encontrados en la literatura, demostrando que en pacientes positivos para LVC la disfunción renal es de gran importancia para auxiliar en el estado clínico.
\end{abstract}

Palabras clave: azotemia, disfunción renal, leishmaniosis

\section{Introdução}

A Leishmaniose Visceral (LV) é uma doença infecciosa grave que acomete as vísceras e que pode ser fatal se não tratada, pois o hospedeiro falha em montar uma resposta protetora eficiente contra o parasita (Silva, 2007). O Brasil é responsável por $90 \%$ dos casos de leishmaniose visceral nas Américas (Silva, 2007), onde a região Nordeste representa o principal foco da doença, com $90 \%$ dos casos, sendo os estados da Bahia, Piauí, Ceará e Maranhão, responsáveis por estes valores (FUNASA, 1999). Em Teresina no ano de 2009, foram registrados 72 casos de LV humana e 3.332 casos de Leishmaniose Visceral Canina (Piauí, 2009; Teresina, 2009). Sendo que estes casos ocorrem principalmente na periferia urbana da cidade, em áreas limítrofes de floresta verde e áreas de pastagens (Werneck et al., 2002).

A doença renal pode ser a única manifestação em cães com LV, podendo progredir de leve proteinúria a síndrome nefrótica, até o estágio de falha renal, sendo a principal causa de morte entre os cães com LV. Assim, é essencial a avaliação da função renal em cães com LV, para um diagnóstico precoce de doença renal, com consequentes benefícios ao cão, o que pode prolongar a sua vida (Solano-Gallego et al., 2009).

Animais com a forma clínica da doença mostram mudanças hematológicas consistentes como uremia, hiperproteinemia e hipergamaglobulinemia, que se apresentam como fortes marcadores para leishmaniose canina (Freitas et al., 2012). Os parâmetros bioquímicos do soro, embora limitados no diagnóstico de LVC, são muito úteis para a avaliação do estado clínico do animal e a extensão das lesões podem dar indicações do prognóstico (Ikeda et al., 2003).

Objetivou-se com esse trabalho constatar as variações nos parâmetros bioquímicos séricos (ureia, creatinina, proteínas totais, albumina e globulina) e alterações na urina de cães positivos, de forma a confirmar a importância dessas avaliações no auxílio ao diagnóstico da doença renal de cães com LVC.

\section{Material e Métodos}

O experimento foi executado no período de janeiro a abril de 2013, no setor de diagnóstico por imagem do Hospital Veterinário Universitário da Universidade Federal do Piauí (HVU/UFPI) do Centro de Ciências Agrárias (CCA) da Universidade Federal do Piauí (UFPI), em Teresina.

Foram utilizados 12 cães, sem raça definida (SRD), machos, jovens (com idade entre 8 meses e 6 anos), de médio porte (peso entre 8 e $15 \mathrm{~kg}$. Foi realizada avaliação clínica, coleta de sangue, punção de medula óssea e linfonodo poplíteo, para diagnóstico de leishmaniose visceral canina, bem como avaliação funcional dos rins através de exame bioquímico do sangue (dosagem de ureia, creatinina, proteínas totais, albumina e globulina) e urinálise. Animais com sorologia e parasitologia negativas para LVC e rins funcionais constituíram o primeiro grupo de 6 animais (grupo controle). E animais com sorologia e parasitologia positiva para LVC com ou sem 
alteração funcional dos rins, e clinicamente assintomáticos constituiram o segundo grupo de 6 animais.

Os exames sorológico e parasitológico de LVC foram realizados no Laboratório de Sanidade Animal (LASAN-UFPI), com o Kit IFI Fiocruz Biomanguinhos, e esfregaços de aspirado de medula óssea e/ou linfonodo poplíteo e, corados com Giemsa e examinados em microscópio de luz convencional, respectivamente. As dosagens bioquímicas foram realizadas utilizando kits comerciais (Labtest Diagnóstica S.A., Lagoa Santa, Minas Gerais, Brasil): ureia, creatinina, proteína total e albumina.

A globulina foi quantificada pela diferença entre a proteína total e a albumina (Alves, 2011). A coleta de urina para urinálise foi feita pela cistocentese de forma asséptica, imobilizando a vesícula urinária por palpação e introduzindo-se percutâneamente uma agulha na porção ventral ou ventrolateral da bexiga, em ângulo de $45^{\circ} \mathrm{em}$ relação à pele e em direção à junção vesicouretral (Ávila et al., 2008).

A análise dos dados obtidos na bioquímica sérica foi feita através de estatística paramétrica em um delineamento de blocos ao acaso e comparação de médias por teste t. Para respostas quantitativas encontradas na urinálise utilizou-se teste não paramétrico de Kruskal-Wallis e para respostas qualitativas tabela de contingência e teste exato de Fisher.

\section{Resultados}

Os níveis de ureia, creatinina, proteínas totais, albumina e globulina estão apresentados na tabela 1. O grupo de animais positivos apresentou maior desvio padrão para os níveis de ureia dosados (57,9 \pm 48,5 mg/dL), onde $33,3 \%$ dos animais apresentaram níveis acima dos valores de referência para cães. A média da concentração de creatinina apresentou-se maior no grupo dos animais positivos $(2,1 \mathrm{mg} / \mathrm{dL})$, mostrando-se mais elevada que os valores de referência. A proteína total mostrou-se aumentada e com maior desvio padrão no grupo dos animais positivos $(7,5$ $\pm 2,8 \mathrm{~g} / \mathrm{dL}$ ) em comparação com os valores de referência. No grupo dos positivos, $50 \%$ dos animais mostraram níveis elevados de proteína total, que está associado com aumento nos níveis de globulinas $(5,65 \pm 2,55 \mathrm{~g} / \mathrm{dL})$ e uma consequente redução na fração de albumina $(1,9$ $\pm 1,0 \mathrm{~g} / \mathrm{dL}$ ) em comparação com os valores de referência para cães. Fato pouco relevante no grupo dos cães negativos, onde na maioria dos animais $(66,7 \%)$ os níveis de proteína total, albumina e globulina mantiveram-se dentro dos valores de referência.

Tabela 1. Parâmetros bioquímicos em cães negativos e positivos para LVC.

\begin{tabular}{lcccc}
\hline Parâmetro & Referência $^{*}$ & Média \pm DP $^{* *}$ & Erro padrão (Sx) & Variação \\
\hline & & & Animais negativos (n=6) \\
Ureia mg/dL & $21-60$ & $56,42 \pm 14,66$ & 14,74 & $35,60-72,50$ \\
Creatinina mg/dL & $0,5-1,5$ & $1,52 \pm 0,46$ & 2,24 & $1,00-2,20$ \\
Proteínas totais g/dL & $5,4-7,1$ & $7,42 \pm 0,75$ & 8,57 & $6,80-8,60$ \\
Albumina g/dL & $2,6-3,3$ & $3,05 \pm 0,65$ & 3,78 & $2,20-3,70$ \\
Globulina g/dL & $2,7-4,4$ & $4,45 \pm 1,49$ & 3,65 & $3,20-6,70$ \\
\hline & & & Animais positivos (n =6) & $19,6-141,90$ \\
Ureia mg/dL & $21-60$ & $57,92 \pm 48,55$ & 8,31 & $0,60-5,80$ \\
Creatinina mg/dL & $0,5-1,5$ & $2,08 \pm 2,08$ & 1,44 & $5,50-9,60$ \\
Proteínas totais g/dL & $5,4-7,1$ & $7,5 \pm 2,79$ & 4,49 & $1,20-3,00$ \\
Albumina g/dL & $2,6-3,3$ & $1,87 \pm 0,95$ & 1,92 & $4,00-8,30$ \\
Globulina g/ dL & $2,7-4,4$ & $5,65 \pm 2,55$ & 3,54 &
\end{tabular}

No exame físico da urina foi possível observar que a coloração padrão para os cães positivos e negativos foi o amarelo. A densidade média da urina mostrou-se menor nos cães positivos $(1028,5)$ que nos cães negativos $(1037,2) . \mathrm{O}$ pH médio da urina dos positivos apresentou-se mais ácido $(6,58)$ em relação ao $\mathrm{pH}$ dos animais negativos $(8,08)$. Em relação ao exame químico $83,3 \%(5 / 6)$ dos cães positivos apresentaram presença de moderada $(30 \mathrm{mg} / \mathrm{dL})$ à marcante (100 mg/dL) de proteínas (albumina) na urina, enquanto nos negativos apenas $16,66 \%$ (1/6) dos 
animais apresentou presença marcante de proteína. Não foi detectado glicose, cetonas ou nitrito em nenhum dos animais dos 2 grupos. Em relação ao exame do sedimento urinário foi possível observar presença mais marcante de cilindros $66,66 \%$ (4/6) e cristais $50 \%$ (3/6) na urina dos cães positivos.

\section{Discussão}

As médias das concentrações de creatinina nos animais positivos mostraram-se aumentados em relação aos valores padrões encontrados em cães saudáveis, bem como os níveis séricos de ureia.

Dentre as alterações clínicas e laboratoriais em cães naturalmente infectados por Leishmaniose, também foram observadas elevações nos níveis de ureia e creatinina no soro de $45 \%$ dos cães com sintomatologia clínica, sendo que a ureia em níveis acima dos valores de referência, juntamente com valores elevados de creatina, configuram um quadro de uremia, podendo significar um provável comprometimento renal (Freitas et al., 2012). Estes resultados são semelhantes aos encontrados por Abreu-Silva et al. (2008), que demonstraram aumentos nos níveis séricos de ureia acima do valore de referência para a espécie canina, em cães naturalmente infectados por Leishmania chagasi.

Alterações encontradas em cães com LVC foram hipoalbuminemia, hiperfosfatemia com consequente hipocalcemia e aumento nos níveis séricos de ureia e creatinina, resultados já amplamente relatados na leishmaniose visceral canina devido à inflamação crônica e doença renal concomitante (Saridomichelakis, 2009, Cortadellas et al., 2006).

Resultados encontrados para dosagem sérica de proteínas totais, albumina e globulinas corroboram com os encontrados por Freitas et al. (2012), onde hiperglobulinemia foi apresentada por $62 \%$ dos animais positivos e hipoalbuminemia foi relatada em $58 \%$ dos animais. Os níveis de proteína total no soro são substancialmente aumentados em cães com LVC e podem atingir níveis acima de $10 \mathrm{~g} / \mathrm{dL}$, devido principalmente a níveis elevados de anticorpos antileishmania (Beta e gama globulinas) relacionados a um quadro sintomático da doença. Assim, o aumento na produção de anticorpos antiLeishmania sp deve ter sido o responsável pela hipergamaglobulinemia e contribuído para a redução na relação entre albumina e globulinas (Silva et al., 2008). A perda seletiva da albumina também pode ocorrer pela urina em decorrência à lesão renal (Grauer, 2005). A frequente proteinúria observada está relacionada à doença glomerular e é reflexo do aumento da permeabilidade capilar glomerular a proteínas do plasma, principalmente a albumina (Soares et al., 2005). Assim como foi observado no presente estudo onde $83,33 \%$ (5/6) dos cães positivos apresentaram presença de moderada $(30 \mathrm{mg} / \mathrm{dL})$ à marcante $(100 \mathrm{mg} / \mathrm{dL})$ de proteínas (albumina) na urina.

\section{Conclusão}

O aumento significativo nos valores de ureia, creatinina e na concentração de proteína da urina dos animais positivos, mostram concordância com os resultados encontrados na literatura. Demonstrando que estas alterações são de grande importância para auxiliar no diagnóstico precoce da doença renal e no estadiamento clínico em pacientes positivos para LVC.

\section{Referências bibliográficas}

Abreu-Silva, A. L., Lima, T. B., Macedo, A. A., Moraes-Junior, F. J., Dias, E. L., Batista, Z. S., Calabrese, K. S., Moraes, J. L., Rebelo, J. M. M. \& Guerra, R. M. S. N. C. 2008. Soroprevalência, aspectos clínicos e bioquímicos da infecção. Revista Brasileira de Parasitologia Veterinária, 17, 197-203.

Alves, G. B. B. 2011. Soro-conversão e avaliação das alterações renais em cães recéminfectados por Leishmania (Leishmania) chagasi. Dissertação. Programa de pósgraduação em ciência animal/ UFPI.

Ávila, A.; Tonini, P. L. J.; Ferreira, P.C.C. 2008. Emergências do trato urinário. In: Santos, M. M.; Fragata, F. S. Emergência e terapia intensiva veterinária em pequenos animais. São Paulo: Roca, 28, 451.

Cortadellas, O., Palacio, M. J. F., Bayón, A., Albert, A. \& Talavera, J. 2006. Systemic hypertension in dogs with leishmaniasis: prevalence and clinical consequences. Journal of Veterinary Internal Medicine, 20, 941-947.

Freitas, J. C. C., Nunes-Pinheiro, D. C. S., Lopes Neto, B. E., Santos, G. J. L., Abreu, C. R. A., Braga, R. R., Campos, R. d. M. \& Oliveira, L. F. 2012. Clinical and laboratory alterations in dogs naturally infected by Leishmania chagasi. Revista da Sociedade Brasileira de Medicina Tropical, 45, 24-29. 
FUNASA. 1999. Boletim epidemiológico Evolução temporal das doenças de notificação compulsória no Brasil de 1980 a 1998. Ministério da Saúde, Fundação Nacional de saúde. Edição especial, ano III

Grauer, G. F. 2005. Canine glomerulonephritis: new thoughts on proteinuria and treatment. Journal of Small Animal Practice, 46, 469478.

Ikeda, F. A., Ciarlini, P. C., Feitosa, M. M., Gonçalves, M. E., Luvizotto, M. C. \& Lima, V. M. F. 2003. Perfil hematológico de cães naturalmente infectados por Leishmania chagasi no município de Araçatuba-SP: um estudo retrospectivo de 191 casos. Clínica Veterinária, 47, 42-8.

Piauí. 2009. Secretaria Estadual da Saúde do Piauí. Coordenação de Vigilância em Saúde Ambiental. Programa de Controle das Leishmanioses. Série histórica de 1998 a 2008. Teresina, PI.

Saridomichelakis, M. N. 2009. Advances in the pathogenesis of canine leishmaniosis: epidemiologic and diagnostic implications. Veterinary Dermatology, 20, 471-489.

Silva, E. B., Fioravanti, M. C. S., Silva, L. A. F., Araújo, E. G., Menezes, L. B., Miguel, M. P. \& Vieira, D. 2008. Característica leucocitária, relação albumina/globulina, proteína plasmática e fibrinogênio de bovinos da raça Nelore, confinados e terminados a pasto. Ciencia Rural, 38, 2191-2196.

Silva, F. S. 2007. Patologia e patogênese da leishmaniose visceral canina. Revista Tropical - Ciencias Agrarias e Biologicas, 1, 20-31.
Soares, M. J. V., Moraes, J. R. E., Palmeira, B. V., Miyazato, L. G. \& Moraes, F. R. 2005. Renal involvement in visceral leishmaniasis dogs. Journal of Venomous Animals and Toxins including Tropical Diseases, 11, 579593.

Solano-Gallego, L., Koutinas, A., Miró, G., Cardoso, L., Pennisi, M. G., Ferrer, L., Bourdeau, P., Oliva, G. \& Baneth, G. 2009. Directions for the diagnosis, clinical staging, treatment and prevention of canine leishmaniosis. Veterinary Parasitology, 165, $1-18$.

Teresina. Gerência de Zoonozes de Teresina. Teresina, PI, 2009 Viana, F.A.B. 2007. Guia Terapêutico Veterinário. 2ed. Gráfica e Editora Cem. 339.

Werneck, G. L., Costa, C. H. N., Walker, A. M., David, J. R., Wand, M. \& Maguire, J. H. 2002. The urban spread of visceral leishmaniasis: clues from spatial analysis. Epidemiology, 13, 364-367.

\section{Article History:}

Received 7 October 2016

Accepted 17 October 2016

Available on line 20 Dezembro 2016

License information: This is an open-access article distributed under the terms of the Creative Commons Attribution License 4.0, which permits unrestricted use, distribution, and reproduction in any medium, provided the original work is properly cited. 